\title{
Entre situations convenues et richesse comique : Les mousquetaires au couvent de Louis Varney
}

\section{Delphine Vincent}

\section{(2) OpenEdition}

\section{Journals}

Édition électronique

URL : http://journals.openedition.org/edl/1151

DOI : $10.4000 /$ edl. 1151

ISSN : 2296-5084

Éditeur

Université de Lausanne

\section{Édition imprimée}

Date de publication : 15 décembre 2016

Pagination : 49-66

ISBN : ISBN 978-2-940331-50-5

ISSN : 0014-2026

\section{Référence électronique}

Delphine Vincent, «Entre situations convenues et richesse comique : Les mousquetaires au couvent de Louis Varney », Études de lettres [En ligne], 4 | 2016, mis en ligne le 15 décembre 2019, consulté le 17 décembre 2020. URL : http://journals.openedition.org/edl/1151; DOI : https://doi.org/10.4000/edl. 1151

\section{(c) Études de lettres}




\author{
ENTRE SITUATIONS CONVENUES \\ ET RICHESSE COMIQUE: \\ LES MOUSQUETAIRES AU COUVENT DE LOUIS VARNEY
}

Louis Varney fut un auteur à succès d'opérettes, qui est tombé de nos jours dans l'oubli. Cet article étudie les procédés de comique musical employés dans son chefd'œuvre, Les mousquetaires au couvent (1880). En s'appuyant sur les écrits théoriques de Baudelaire et de Bergson, cet essai souligne la variété du comique dans cette opérette: verbal, gestuel, mécanique, hypertrophie musicale, détournement musical, jeu avec des situations musicales topiques, superposition de niveaux de lecture. La richesse des réponses musicales proposées par Varney est remarquable et rappelle la valeur d'un artiste qui souffre de la mauvaise réputation de l'opérette française - dépolitisée et passant pour un pur divertissement - des années 1880 .

Si les opérettes de Jacques Offenbach sont très connues et jouées, il n'en va plus de même des œuvres légères écrites en France par la génération suivante de compositeurs. En effet, il est difficile de citer des titres encore montés de nos jours, et ce malgré l'immense succès rencontré par nombre de ces pièces lors de leur création. C'est le cas des opérettes de Louis Varney, tombées dans un oubli injustifié au vu de leur qualité.

Né en 1844 à La Nouvelle-Orléans, Louis Varney est le fils du compositeur et chef d'orchestre Alphonse Varney (1811-1879), qui avait été le directeur du théâtre des Bouffes-Parisiens lorsqu'Offenbach y était actif (1857-1865). Louis vient au monde alors que son père dirige la saison d'opéra français à La Nouvelle-Orléans. Ce dernier assure sa formation, le prédestinant à une carrière dans les genres légers. Il débute, en effet, comme chef au Théâtre de l'Athénée-Comique à Paris (1876), pour lequel il compose la même année une opérette en un acte, Il signor Pulcinella, qui fut un grand succès. Il écrit alors surtout des musiques de 
revues. Ce n'est qu'après la mort de son père, en 1879, qu'il devient un compositeur prolifique d'opérettes. Sa première œuvre, Les mousquetaires au couvent, est créée aux Bouffes-Parisiens, le 16 mars 1880. Pendant vingt ans, il produit deux opérettes par an, dont Fanfan-la-tulipe (1882) et Babolin (1884), ainsi que des ballets. Au cours de ses dernières années, il compose peu à cause d'une maladie qui l'oblige à résider à Bagnèresde-Bigorre dans les Pyrénées. Rentré à Paris le 19 août 1908, il y meurt le lendemain ${ }^{1}$.

Malgré cet œuvre considérable, la seule opérette de Varney qui est restée au répertoire est Les mousquetaires au couvent. Elle avait rencontré, dès sa création, un immense succès en France et à l'étranger, circulant très rapidement en traduction anglaise à Londres (1880), russe à SaintPétersbourg (1881), allemande à Vienne (1881), espagnole à Barcelone (1881) et italienne à Rome (1883) ${ }^{2}$. Reprise régulièrement, d'abord aux Bouffes-Parisiens, puis partout à Paris jusqu'au début de la Première Guerre mondiale, cette opérette est toujours jouée dans les pays francophones, en témoigne sa reprise à l'Opéra de Lausanne en $2013^{3}$. Cette popularité s'explique peut-être par une intrigue typique du genre de cape et d'épée qui, de nos jours, n'a pas perdu son attrait, mais aussi par la qualité certaine de la partition livrée par Varney.

\section{Une intrigue conventionnelle}

Les mousquetaires au couvent se déroule en Touraine, sous le règne de Louis XIII. Marie et Louise, les nièces du gouverneur de Touraine, sont pensionnaires au couvent des Ursulines. Marie est amoureuse d'un mousquetaire du roi, Gontran, dont elle est aimée en retour. Le rideau s'ouvre à Vouvray dans l'auberge "Au Mousquetaire gris». Gontran avoue son amour pour Marie à ses amis, le mousquetaire Narcisse de Brissac et son précepteur l'abbé Bridaine. Les deux mousquetaires

I. Voir K. Gänzel, A. Lamb, "Varney, Louis».

2. J. Heinzelmann, «Louis Varney».

3. Cet article propose une version élaborée des deux conférences, l'une à l'Université de Lausanne ("L'habit ne fait pas le moine: Les mousquetaires au couvent de Louis Varney", 11 décembre 2013) et l'autre au Théâtre Municipal de Lausanne pour Forum Opéra ("Les mousquetaires au couvent de Louis Varney», 10 décembre 2013), données à l'occasion de cette production. 
souhaitent enlever Marie, mais Bridaine s'y oppose et propose de plaider la cause des amoureux auprès du gouverneur. Toutefois, ce dernier vient annoncer à ses nièces qu'elles doivent prendre le voile pour servir la politique du cardinal de Richelieu. Brissac et Gontran profitent du fait que le gouverneur est aidé par deux moines errants pour les dépouiller et se rendre à leur place au couvent des Ursulines, qui servira de cadre aux deux actes suivants.

Dès qu'ils sont arrivés, Gontran se fait reconnaître de Marie, alors que Brissac sympathise avec Louise et détonne par son vocabulaire militaire coloré. Cependant, Bridaine persuade Marie d'écrire à Gontran qu'elle ne l'aime pas. L'abbé reconnaît alors Brissac et Gontran sous la robe des moines errants, mais choisit de ne pas les dénoncer. Toutefois, il décide de montrer la lettre de Marie à Gontran, qui n'est pas dupe du stratagème entrepris par Bridaine pour les séparer. Pendant ce temps, Brissac profite du copieux déjeuner et, ivre, livre un sermon sur l'amour - plus charnel que divin - suscitant un immense scandale, correspondant au final du deuxième acte.

Au début du troisième acte, Brissac, enfin dégrisé, aide Gontran dans ses projets d'enlèvement. C'est alors que Louise demande à être de la partie. Au moment où les quatre acolytes prennent le large, Bridaine surgit, suivi de peu par le gouverneur. Un retournement in extremis de situation permet à Gontran et à Brissac d'échapper à leur châtiment: les moines dont ils avaient volé les habits étaient en fait de faux moines cherchant à assassiner le cardinal de Richelieu. Grâce à eux, les faussaires ont été arrêtés: en récompense, Gontran épouse Marie et Brissac obtient la main de sa sœur Louise. Tout est bien qui finit bien!

Les mousquetaires au couvent propose une intrigue bien convenue pour un genre qui passe, en décrivant de manière acide le monde de l'époque, pour être décapant. La raison de ce choix est simple: après la chute du Second Empire en 1870, l'opérette offenbachienne, extrêmement critique vis-à-vis de la société contemporaine à travers des satires sans concessions de ses mœurs, ne rencontre plus la faveur du public. Ce dernier préfère alors les opérettes moins piquantes et plus romantiques. Qui plus est, c'est également à cette période - dès la fin des années 1860 et dans les années 1870 - que l'opérette viennoise se développe. Cette tendance est, elle aussi, exempte de critique sociale frénétique.

Dans Les mousquetaires au couvent, on ne retrouve donc ni les satires $\mathrm{du}$ quotidien de la vie sociale, ni les parodies de la mythologie, deux 
types de sujets prisés par Offenbach. Afin d'éviter une charge critique trop élevée, les librettistes de Varney, Paul Ferrier et Jules Prével, recourent à une comédie-vaudeville, L'habit ne fait pas le moine, d'Amable Vilain de St-Hilaire et Paul Duport, créée à Paris (au théâtre national du Vaudeville, le 18 août 1835) avec des musiques d'Alexandre Doche, Thénard (Etienne Perrin) et Alphonse This. Suite au succès phénoménal des romans historiques de Walter Scott au début du XIX ${ }^{\mathrm{e}}$ siècle et à la naissance du genre en France avec Cinq-Mars (1826) d'Alfred de Vigny, le roman et le drame historiques s'implantent parmi les genres dominants en France. L'opéra n'est pas en reste, Eugène Scribe tire des livrets des ouvrages de l'écrivain écossais dès les années 1820. C'est dans ce cadre que L'habit ne fait pas le moine est écrit. Par la suite, le genre "de cape et d'épée" s'établira comme un succès populaire grâce aux feuilletons, avec notamment Les Trois mousquetaires (1844), Vingt ans après (1845) et Le Vicomte de Bragelonne (1848) d'Alexandre Dumas et Le bossu (1855) de Paul Féval. Avec Les mousquetaires au couvent, Varney s'insère dans ce courant et ne vise qu'au divertissement, contrairement aux opérettes d'Offenbach, son illustre prédécesseur. Lancienneté de la source, ainsi que le genre de cape et d'épée, évitent tout lien avec la situation sociale et politique contemporaine. Il s'agit probablement de l'une des raisons pour lesquelles cette phase ultérieure de l'opérette est moins considérée de nos jours, voire regardée avec condescendance.

\section{Style musical}

Si le livret des Mousquetaires au couvent lorgne vers la première partie $\mathrm{du} \mathrm{XIX}^{\mathrm{e}}$ siècle, il n'en est rien de sa mise en musique. Nous sommes, naturellement, bien loin des vaudevilles dans lesquels l'action se déroulait durant les dialogues de la pièce qui était agrémentée de chansons (en général des timbres, c'est-à-dire des mélodies déjà connues et indiquées "sur l'air de»). Varney se positionne entre l'écriture mécanique à la Offenbach et le lyrisme de Johann Strauss II. En effet, l'«Ouverture» des Mousquetaires au couvent est très nettement redevable à Offenbach par son élan frénétique, tout comme la "Chanson villageoise» entonnée par Simone, qui servira de conclusion à l'œuvre. Cependant, Varney écrit également de nombreuses valses ou romances dont certaines lors de situations pathétiques, par exemple lorsque Gontran doute de l'amour 
de Marie ("Il serait vrai! Ce fut un songe»). Ces différences d'écriture montrent à quel point Varney a assimilé les deux styles et comment il est capable de varier son écriture selon les situations offertes par son livret.

Cependant, toutes les valses des Mousquetaires au couvent n'ont pas ce caractère lyrique sentimental, typique de l'opérette viennoise. Varney exploite parfois cette danse pour créer une situation comique. Il n'hésite pas à ridiculiser des situations religieuses en leur donnant un accompagnement valsé, ô combien inadéquat dans un couvent. Lors de la relecture de l'examen de conscience écrit par les pensionnaires (les couplets «Mon Père, je m'accuse»), le mouvement de valse correspond au peu de sérieux que les jeunes filles mettent à le rédiger (elles ont décidé d'écrire toutes le même!). Ce choix n'est pas innocent et témoigne d'un ancrage dans les problématiques contemporaines, bien que moins acerbe que celui des opérettes d'Offenbach, en offrant un écho au climat extrêmement anticlérical régnant dans la France de 1880. Rappelons-nous que Baudelaire a souligné que "l'homme mord avec le rire» ${ }^{4}$.

Dès lors, il convient de relativiser un jugement séparant trop nettement deux styles d'écriture antithétiques. Varney joue avec succès des possibilités offertes par ces deux types, intégrant, en outre, le détournement comique de leurs conventions. C'est bien dans la variété des réponses musicales que Varney a su apporter aux situations comiques traditionnelles de son livret que réside l'intérêt des Mousquetaires au couvent. En regardant de plus près les mécanismes de la production du comique en musique, on se rend compte qu'il met un soin particulier à ne pas faire rire le spectateur gratuitement. Pour ce faire, il varie les procédés de manière remarquable.

\section{Comique verbal et gestuel}

Malgré l'accent que nous voulons placer sur l'étude du comique musical, il convient, tout d'abord, de souligner qu'une bonne partie du comique passe dans Les mousquetaires au couvent par les dialogues qui s'insèrent entre les numéros chantés. Notons que la partition indique

4. Ch. Baudelaire, «De l'essence du rire et généralement du comique dans les arts plastiques", Le Portefeuille, 8 juillet 1855; cité d'après Ch. Baudelaire, Baudelaire journaliste, p. 199. 
"opéra comique» et non "opérette", décrivant par ce terme un ouvrage long, avec des dialogues parlés et une musique relativement complexe. N'oublions pas que, à la fin du XIX ${ }^{\mathrm{e}}$ siècle, les frontières entre les genres comiques sont beaucoup plus floues quauparavant et que l'«opéra comique" n'est plus lié à une situation institutionnelle.

Le comique verbal joue abondamment avec les possibilités offertes par l'usurpation d'identité des deux mousquetaires. Brissac est dépeint comme un personnage qui, bien que déguisé en moine, est incapable de ne pas se trahir par l'emploi d'un vocabulaire non approprié ou de détourner le sens des paroles des sœurs (pour des mots tels que «discipline» ou "passer en revue»). Il entre donc dans la catégorie de personnages comiques décrite par Henri Bergson en ces termes:

Mais le moyen le plus usité de pousser une profession au comique est de la cantonner, pour ainsi dire, à l'intérieur du langage qui lui est propre. On fera que le juge, le médecin, le soldat appliquent aux choses usuelles la langue du droit, de la stratégie ou de la médecine, comme s'ils étaient devenus incapables de parler comme tout le monde. D'ordinaire, ce genre de comique est assez grossier. Mais il devient plus délicat, $[\ldots]$ quand il décèle une particularité de caractère en même temps qu'une habitude professionnelle 5 .

Outre le comique verbal, le jeu d'acteur, principalement des rôles comiques, tient également une part importante, notamment le comique gestuel. Il est naturellement difficile d'estimer quels étaient les choix effectués lors de la création et des représentations suivantes. Il en va de même pour le texte parlé qui peut être abondamment varié ou coupé selon les occasions. S'il s'agit de deux composantes fondamentales des genres comiques, il est important de se rappeler que ces dimensions sont sujettes à des modifications (sans entrer dans la question épineuse des traductions), sans enlever, toutefois, à leur portée comique. L'adaptation de ces deux paramètres constitue une constante des genres comiques, que l'on craint moins de modifier car ils sont considérés comme plus légers, et non une spécificité des Mousquetaires au couvent.

5. H. Bergson, Le rire, p. 137. 


\section{Situations topiques du comique musical}

Le comique n'est, bien évidemment, pas cantonné à la parole et aux gestes dans le théâtre musical. Commençons par souligner que certaines situations sont devenues des classiques du numéro chanté comique et qu'elles se retrouvent, infiniment variées, dans l'histoire de l'opéra comique (entendu dans son sens générique et non de genre). Les principales adaptations par rapport à la source du livret, la comédie-vaudeville L'habit ne fait pas le moine, sont d'ailleurs effectuées afin de ménager ce type de situations convenues. A ce sujet, Bergson a souligné que:

Quand une scène comique a été souvent reproduite, elle passe à l'état de "catégorie» ou de modèle. Elle devient amusante par elle-même, indépendamment des causes qui font qu'elle nous a amusés ${ }^{6}$.

L'intrigue des Mousquetaires au couvent permet d'exploiter de manière topique la rencontre entre les mondes militaire et religieux, à commencer par la prière des faux pèlerins, entrecoupée de retours à leurs préoccupations véritables. Cette situation n'est en rien nouvelle: dans Le comte Ory (1828) de Gioachino Rossini, Ory et ses compagnons, déguisés en pèlerines afin de séduire la belle comtesse Adèle, oscillent entre une chanson à boire et une prière pour donner le change.

Dans le final du premier acte des Mousquetaires au couvent, l'arrivée des faux pèlerins de Palestine interrompt la fête en cours. L'assemblée se joint alors à leur prière dans une parodie de choral: "Le front dans la poussière, amis prosternons-nous! Ecoutons à genoux leur fervente prière. Et vous saints pèlerins le ciel soit avec vous!» Puis, les faux pèlerins (Gontran et Brissac) chantent leurs couplets «Nous venons de la Palestine» avec des ponctuations de l'assemblée, qui rappellent le chant responsorial. Afin de donner crédit à leur usurpation, ils lancent quelques formules liturgiques en latin: «Pax Domini sit vobiscum», "Benedicat vos Dominus", "Semper cum spiritu tuo" et "Agamus Deo gratias». Gontran, à l'évocation de se rendre au couvent dans lequel demeure Marie, s'oublie et chante «O doux espoir! Je vais donc te revoir! C'est un moment bien doux». Brissac le remet à l'ordre par un "Silence! à genoux" qui enclenche à nouveau la prière du chœur ("Le front dans la poussière»). Toutefois, Brissac, retrouvant le mousquetaire Rigobert,

6. Ibid., p. 73 . 
reprend son langage habituel de militaire et règle le déroulement des opérations futures. Son chant se superpose donc à celui de l'assemblée qui reprend sa prière. Finalement, les deux faussaires concluent par une ultime formule latine "Pax Domini sit semper vobiscum ", avant de sortir de scène. Varney crée ainsi une sonorisation relativement conventionnelle d'un lieu commun, mais diablement efficace.

Le choc entre les mondes militaire et religieux est également au cœur du final du deuxième acte. Brissac, ivre suite à un déjeuner copieux, se propose de prêcher. Il choisit comme premier sujet l'abstinence, mais prêche finalement sur l'amour, qu'il ne limite pas à l'amour divin, glissant sur l'amour charnel («Moi si j'aime tous mes prochains, j’aime aussi toutes mes prochaines»). Le refrain de ses couplets énonce son credo ("Aimons-nous, aimons-nous donc! Tel est mon prêche, qui n'aime pèche»), qui suscite le commentaire horrifié de l'assemblée: "Il prêche une étrange morale. Juste Ciel! Quel affreux scandale!» Alors que dans L'habit ne fait pas le moine le rideau tombait rapidement, les librettistes décident d'exploiter pleinement ce coup de théâtre et créent un parfait final d'acte. D'ailleurs, Bergson a noté que:

Chacun sait avec quelle facilité la verve comique s'exerce sur les actes sociaux à forme arrêtée, depuis une simple distribution de récompenses jusqu'à une séance de tribunal. Autant de formes et de formules, autant de cadres tout faits où le comique s'insérera ${ }^{7}$.

Dans ce cas, il s'agit naturellement d'un comique créé par les dérapages de Brissac dans le cadre établi du prêche. En outre, ses couplets, avec le refrain "Aimons-nous", font penser à l'invocation à Venus de La belle Hélène (1864) d'Offenbach, dans laquelle la protagoniste affirme: "Il nous faut de l'amour". Là encore, nous retrouvons l'idée d'une scène comique modèle.

La présence des mousquetaires permet également d'intégrer leur chanson "La ronde du beau mousquetaire", chantée par Simone. Elle contient naturellement un "rantanplan», ainsi qu'un roulement vocal sur "rrr», afin d'évoquer le tambour militaire. L'onomatopée est un procédé traditionnel du comique et cette situation est également topique. Elle apparaît, notamment, dans La fille du régiment (1840) de Gaetano Donizetti et La forza del destino (1862/1869) de Giuseppe Verdi.

7. Ibid., p. 35 . 
Un autre topos figure à la fin des Mousquetaires au couvent, alors que les quatre protagonistes tentent de s'échapper grâce à une échelle ("Quintette de l'échelle»). Ici, la situation, et non le type d'écriture musicale, fait penser au Barbiere di Siviglia (1816) de Rossini. Toutefois, il s'agit aussi d'une situation typique de la littérature de cape et d'épée: l'enlèvement d'une jeune fille d'un couvent, qui souligne à quel point le livret est ancré dans un genre populaire. Il s'agit, cependant, d'une addition des librettistes par rapport à L'habit ne fait pas le moine, qui présentait un agencement un peu différent du troisième acte, bien que l'échelle y soit déjà mentionnée.

\section{Perte du sens et mécanicité}

Outre les situations comiques classiques du théâtre musical, les techniques d'écriture peuvent également susciter le rire. Certains procédés musicaux sont construits de manière à faire perdre le sens sémantique de la parole, comme cela arrive fréquemment chez Offenbach. Une de ces techniques consiste à accentuer une syllabe particulière d'un mot donné, afin de générer une perte de sens confinant à l'absurde. Dans Les mousquetaires au couvent, l'abbé Bridaine détache et accentue une seule syllabe d'un mot dans «Eh! oui c'est moi l'abbé Bridaine». Le résultat est particulièrement comique, car c'est son nom qu'il étire démesurément «Je suis l'abbé Bridai-ai-ai-ne». Il s'agit de ses couplets de présentation, qui sont typiques des airs d'entrée des personnages comiques dans une opérette. En effet, la forme strophique (couplet-refrain) est souvent employée dans les genres comiques, car la complexité musicale y est moins recherchée que dans les genres sérieux. L'abbé Bridaine entre en scène de manière classique pour un personnage comique: en chantant des couplets dans lesquels il s'auto-présente au public (il proclame d'ailleurs: "Je suis l'abbé Bridaine, un si bon garçon qu'on peut sans façon me mettre en chanson") et avec un langage musical qui contribue à sonoriser son statut comique. Bridaine semble ainsi être en contradiction avec la remarque de Baudelaire: "Ils [les artistes] savent que tel être est comique, et qu'il ne l'est qu'à condition d'ignorer sa nature ${ }^{8}$.

8. Ch. Baudelaire, Baudelaire journaliste, p. 217. 
Le même procédé se retrouve dans les couplets de Simone, "A la porte des révérends", dans lesquels elle allonge "pi-toy-a-ble», puis lors de la seconde strophe "effr-oy-a-ble", ce qui donne un tour comique à son récit qui, sinon, pourrait être pathétique (elle évoque les faux moines de l'auberge qui "crient aux assassins»). Ces similitudes d'écriture soulignent bien que, comme l'a remarqué Bergson:

Le personnage comique est un type. Inversement, la ressemblance à un type a quelque chose de comique?

Varney étend parfois ce procédé - le morcellement des mots par syllabes, qui perdent ainsi leur dimension sémantique et deviennent pur effet comique - en le combinant avec le côté mécanique du discours musical typique de Rossini. D’ailleurs, Bergson a souligné la part importante que tiennent la raideur mécanique et la répétition dans le comique:

Là où il y a répétition, similitude complète, nous soupçonnons du mécanique fonctionnant derrière le vivant. [...] Cet infléchissement de la vie dans la direction de la mécanique est ici la vraie cause du rire ${ }^{10}$.

C'est le cas de l'air de Brissac «Gris, suis-je gris» qu'il chante après avoir copieusement déjeuné et totalement ivre. La situation fait, notamment, penser à "Dans ce lieu solitaire", chanté par Raimbaud dans Le comte Ory de Rossini. Ces deux morceaux ont en commun la liste du festin à venir (Le comte Ory) ou juste consommé (Les mousquetaires au couvent). Dans ce cas, une partie des procédés d'écriture employés par Varney renvoient à une écriture comique ancienne, justement celle de Rossini. En effet, on retrouve le côté mécanique du discours rossinien dans la partie rapide de l'air (Allegro moderato, "De la chère qu'on m’a donné»). En outre, le catalogue est typique des airs comiques, notamment dans la tradition de l'opera buffa.

Le mécanique peut également se manifester par la duplication parodique d'un événement. Dans Les mousquetaires au couvent, les pensionnaires doivent écrire, au début du deuxième acte, une dictée "Donc Rébecca sa cruche pleine» (il s'agit d'un ajout par rapport à L'habit ne fait pas le moine). Elles ponctuent la lecture de sœur Opportune par l'onomatopée comique "ton ton et tontaine», soulignant ainsi à quel point elles

9. H. Bergson, Le rire, p. 113.

Io. Ibid., p. 26. 
trouvent l'exercice barbant. Lorsqu'il s'agit, peu après, de rédiger leur examen de conscience, les jeunes filles décident d'écrire toutes le même sous la dictée de Louise. La proximité des deux numéros suscite le rire, tant le détournement est évident. D’ailleurs, Bergson a remarqué que:

On devine que les artifices usuels de la comédie, la répétition périodique d'un mot ou d'une scène, l'interversion symétrique des rôles, le développement géométrique des quiproquos, et beaucoup d'autres jeux encore, pourront dériver leur force comique de la même source, l'art du vaudevilliste étant peut-être de nous présenter une articulation visiblement mécanique d'événements humains tout en leur conservant l'aspect extérieur de la vraisemblance, c'est-à-dire la souplesse apparente de la vie ${ }^{11}$.

\section{Hypertrophie et détournements musicaux}

Le théâtre musical a développé un autre moyen pour faire rire le spectateur: souligner excessivement le sens de chaque parole, au point de perdre la cohérence du discours musical. Ce procédé est particulièrement évident dans "Gris, suis-je gris", peut-être parce que Varney a eu plus de temps pour le composer. En effet, ce numéro ne figure pas dans la version originale, écrite dans l'urgence suite à une commande de Louis Cantin, directeur des Bouffes-Parisiens (Varney n'avait, d'ailleurs, pas eu le temps d'achever la partition, qui fut complétée - fin du troisième acte et entractes - par Achille Mansour). Varney révisa son œuvre pour la saison suivante (2 septembre 1880). Il modifia alors principalement le rôle de Brissac joué précédemment par un acteur qui chantait, le comédien Frédéric Achard qui possédait une voix agréable. Dans cette versionlà, la partie de baryton comprenait des parties d'ensemble importants, mais peu de soli. Lorsque Varney réécrit la partition pour le baryton star, Louis Morlet, il ajoute deux airs, "Gris, suis-je gris" et "Pour faire un brave mousquetaire». Ces deux pièces, écrites sur des sujets convenus, arrêtent le déroulement dramaturgique et exhibent ainsi clairement leur statut d'ajout. C'est cette partition qui est devenue la version définitive de l'œuvre.

II. Ibid., p. 27 sq. 
Varney utilise dans "Gris, suis-je gris» un certain nombre de procédés qui imitent le discours désordonné d'un homme ivre. Il débute par un récitatif qui est un chef-d'œuvre comique. Tout d'abord, il s'agit d'un détournement parodique très réussi du traditionnel récitatif accompagné réservé aux personnages de haut niveau social dans des situations tragiques. Après une première phrase à la dominante si bémol, la ponctuation de l'orchestre dirige le chanteur vers la tonique mi bémol. Brissac, qui est ivre, éprouve quelques difficultés à y parvenir. En effet, la phrase "Mais la faute est aux bonnes sœurs" est morcelée par des silences: à chaque fois, Brissac part d'un $\mathrm{sol}^{3}$ et opte pour un intervalle plus grand (successivement $s i^{3}-d o^{4}-$ ré $^{4}-m i$ bémol ${ }^{4}$ ), faute de meilleure idée musicale, mais aussi parce qu'il ne sait plus très bien dans quelle tonalité il évolue et peine à atteindre la tonique mi bémol. Outre les silences entre les mots qui indiquent les difficultés de Brissac à trouver ses mots, Varney utilise également des césures à l'intérieur des mots comme dans le cas de "aux bon-» "-nes sœurs", séparés par un silence des plus absurdes.

Brissac retrouve ensuite un débit de parole normal, lorsqu'il évoque les douceurs dont le comblèrent les bonnes sœurs et auxquelles il fut très sensible. Il opte alors pour un langage lyrique, correspondant aux mots «douceurs» et «sensibles». Il bascule, par réflexe, dans un chant de type courtois, totalement inapproprié aux bonnes sœurs dont il est question! On constate l'ornement sur "comblèrent de», puis la cadence sur "sensi-ble", qui n'a aucune logique musicale, si ce n'est celle de l'habitude galante. Cette attitude implique que l'on retrouve le morcellement des mots, par l'arrêt sur certaines syllabes, ici sur sen-si-ble». Dans la section lente qui débute l'air ("Ah! ah! quel déjeuner j’ai fait»), Varney place un ritardando comique sur «stu-pé-fait», alors que Brissac annonce qu'il demeure stupéfait de la perfection de son déjeuner. Le discours musical est suspendu pour décrire son abasourdissement. Dans la section rapide qui suit, il évoque tous les mets qu'il a mangés et les crus qu'il a bu pour les accompagner. Il mentionne notamment « une truite saumonée avec un marsala très vieux". A ce moment-là, Varney insère entre les phrases musicales une répétition de "très vieux", parlé et non chanté, par le même Brissac qui fait écho à ses propos, comme pour surenchérir, et souligne le soliloque d'un homme saoul. Cet air est donc basé sur l'hypertrophie, l'un des procédés usuels du comique musical, et réalisé de main de maître par Varney. Son immense succès n'est en rien usurpé! 
Le comique peut aussi passer par un effet opposé au soulignement des détails du texte, c'est-à-dire par le détournement du sens des paroles, réalisé grâce à une musique qui n'en épouse pas la signification. Dans ce cas, c'est le contraste entre les deux éléments qui provoque le rire. Lors de l'arrivée du gouverneur, les villageois sont déçus de devoir arrêter leur fête ("Maudit soit le gouverneur»), comme le leur enjoint Pichard: "Alerte! Le gouverneur est sur mes pas. Que la danse s'arrête! Respect à Monseigneur». A la suite de Simone, le chœur reprend alors l'exclamation:

Maudit soit le gouverneur qui vient troubler notre fête. Quel ennui! Devant lui faut-il que la fête cesse. Son retour, sur ce jour jette un voile de tristesse. Mais tout bas sur ses pas, tout en feignant l'allégresse, chacun dit: quel malheur lorsquarrive le Gouverneur.

La déclamation se déroule sur une valse en complet désaccord avec le sens des paroles, mais qui correspond au désir intérieur des villageois de continuer à faire la fête. Ce chœur ne figure pas dans L'habit ne fait pas le moine, ce qui n'est pas étonnant, vu que le comique naît de la juxtaposition d'un texte et d'une musique contradictoires, créant ainsi un numéro relativement compliqué.

Outre le détournement du sens des paroles, la musique peut également ne pas être appropriée à la situation en train de se dérouler. Varney joue à plusieurs reprises avec l'inadéquation d'une musique de danse profane, la valse, dans un couvent pour susciter le rire des spectateurs, comme dans les couplets, «Mon Père, je m'accuse», des pensionnaires.

\section{Superposition comique de niveaux de lecture}

Au-delà du fait de coller à une situation ou de la détourner, la musique offre la possibilité de superposer des réactions simultanées diversifiées. Pour ce faire, elle peut opposer des musiques de types différents ou faire partager le même discours musical à tous les personnages, même s'ils expriment des sentiments divergents. C'est cette solution que Varney choisit pour le trio "Parle! Explique-toi!", dans lequel Brissac et Bridaine essaient de soutirer à Gontran le secret qu'il leur cache. Lorsque ce dernier avoue que c'est une femme qui est la cause de son état, Varney enchaîne sur la réaction de stupeur des deux autres qui 
se déroule sur fond de valse. Si cette musique de danse correspond à la déclaration lyrique de l'amour de Gontran pour Marie ("Amoureux de toute mon âme et si le ciel trahit ma flamme, puissé-je mourir sous ses yeux»), elle ne convient pas aux propos des deux autres personnages. Elle en devient même ridicule car, pour former un fragment complet de phrase musicale, il faut que l'auditeur associe les éléments chantés par les deux hommes. En effet, Brissac et Bridaine adoptent la répartition classique des instruments dans la valse - certains énonçant le premier temps, d'autres les deux autres - même si eux se répartissent le matériel par mesure (Bridaine à la première mesure "une femme», Brissac sur la deuxième "une femme", Bridaine sur la troisième "une femme", Brissac sur la quatrième "une femme», etc.). La structure de la valse permet de créer différents niveaux - sérieux ou comique - simultanés suivant les personnages. La situation ainsi générée est relativement complexe, étant donné qu'elle superpose deux niveaux de lecture différents.

En outre, la stupeur est dépeinte par un arrêt comique sur un seul mot ou presque ("une femme»). La répétition est évidemment un élément essentiel de la production du comique, comme Bergson l'a noté: «un des procédés usuels de la comédie classique, la répétition" ${ }^{12}$. Ces éléments rappellent Offenbach, notamment sa parodie de concertato de stupeur avec «L'homme à la pomme» dans La belle Hélène. Enfin, alors que les deux hommes essaient de découvrir l'identité de l'amour de Gontran, ils énumèrent des possibilités: "une comtesse, une duchesse, une princesse, une drôlesse». La liste, nous l'avons vu, est également un élément traditionnel du comique, toutefois elle permet aussi de susciter le rire grâce à la répétition mécanique des hypothèses de Brissac par Bridaine. Celui-ci répète machinalement "une drôlesse", qui n’appartient clairement pas à son vocabulaire ecclésiastique, mais qui fait bel et bien partie du langage coloré des mousquetaires. Il se reprend alors: "Eh là! Que me faitesvous dire là?", soulignant par là même aux spectateurs l'incongru de son intervention précédente. Bergson a, d'ailleurs, individualisé ce type de réparties comme un ingrédient typique du comique:

Se laisser aller, par un effet de raideur ou de vitesse acquise, à dire ce qu'on ne voulait pas dire ou à faire ce qu'on ne voulait pas faire, voilà, nous le savons, une des grandes sources du comique. C'est

I2. Ibid., p. 55. 
pourquoi la distraction est essentiellement risible. [...] Il n'y a d'essentiellement risible que ce qui est automatiquement accompli ${ }^{13}$.

La superposition de niveaux de lecture ne concerne pas uniquement le rapport entre les voix, mais peut aussi se produire entre les chanteurs et l'orchestre. Dans les couplets chantés par Gontran et Brissac déguisés en pèlerins ("Nous venons de la Palestine»), les voix chantent dans une écriture conforme à la situation: le chœur ponctuant les phrases des pèlerins en reprenant leur dernier mot. Toutefois, l'orchestre ne se limite pas à doubler les voix: des traits très rapides et légers s'insèrent dans le discours, donnant à la scène un caractère comique. Il en va de même dans la dictée "Donc Rébecca sa cruche pleine». Si sœur Opportune chante sur le ton déclamatoire approprié à l'exercice, l'orchestre présente un motif léger qui vient souligner la dissipation des pensionnaires à ce moment-là.

\section{Conclusion}

Ce tour d'horizon des procédés musicaux comiques dans Les mousquetaires au couvent permet de prendre conscience du fait que, si Varney reçoit un livret conventionnel, il en fait son miel en variant son écriture tout au long de l'œuvre. Par là même, nous nous rendons compte qu'il connaît très bien l'opéra et l'opérette de son siècle et compose de la musique de valeur, même si souvent son écriture n'est pas extrêmement complexe, comme le veut d'ailleurs le genre. Cette connaissance, alliée à une maîtrise parfaite de son art, lui permet d'exploiter des langages musicaux comiques variés allant de Rossini à Offenbach. Il peut ainsi choisir différents procédés de mise en musique, suivant les types de comique présentés par le livret. En outre, Varney ne se contente pas de souligner ces moments, mais crée souvent des situations propices au rire grâce au décalage qu'il introduit dans sa musique par rapport aux paroles ou aux événements en train de se dérouler. Si Varney produit une opérette française typique des années 1880 - relativement peu politisée et critique - c'est bien la richesse des réponses musicales qu'il propose

I3. Ibid., p. 85 et 111. 
face aux situations comiques figurant dans le livret qui a permis aux Mousquetaires au couvent de rester, à juste titre, au répertoire.

\author{
Delphine Vincent \\ Université de Fribourg
}




\section{BIBLIOGRAPHIE}

Baudelaire, Charles, Baudelaire journaliste. Articles et chroniques, éd. Alain Vaillant, Paris, Flammarion, 2011.

Bergson, Henri, Le rire. Essai sur la signification du comique, Paris, Quadrige/Presses Universitaires de France, 2012 (1900).

Gänzel, Kurt, Lamb, Andrew, "Varney, Louis", in The New Grove Dictionary of Opera, ed. by Stanley Sadie, John Tyrrell, Grove Music Online (http://www.oxfordmusiconline.com/subscriber/ article/grove/music/O905429>) (23.03.2015).

Heinzelmann, Josef, "Louis Varney", in Pipers Enzyklopädie des Musiktheaters, hrsg. von Carl Dahlhaus und Sieghart Döhring, München/Zürich, Piper, 1997, p. 378-380.

Lamb, Andrew, "Varney, Louis", in The New Grove Dictionary of Music and Musicians, ed. by Stanley Sadie, John Tyrrell, Grove Music Online (http://www.oxfordmusiconline.com/subscriber/article/ grove/music/29057>) (23.03.2015). 
